\title{
J oint Optimization of Mobile Charging and Data Gathering for Wireless Rechargeable Sensor Networks
}

\author{
Xianzhong Tian, Jiacun He, Yuzhe Chen and Yanjun Li* \\ School of Computer Science and Technology, Zhejiang University of Technology \\ Hangzhou 310023 - China \\ [e-mail: txz@zjut.edu.cn,372044849@qq.com,yzchen0618@gmail.com, yjli@zjut.edu.cn] \\ *Corresponding author: Yanjun Li \\ Received November 5, 2018; revised December 26, 2018; accepted January 30, 2019; \\ published July 31, 2019
}

\begin{abstract}
Recent advances in radio frequency (RF) power transfer provide a promising technology to power sensor nodes. Adoption of mobile chargers to replenish the nodes' energy has recently attracted a lot of attention and the mobility assisted energy replenishment provides predictable and sustained power service. In this paper, we study the joint optimization of mobile charging and data gathering in sensor networks. A wireless multi-functional vehicle (WMV) is employed and periodically moves along specified trajectories, charge the sensors and gather the sensed data via one-hop communication. The objective of this paper is to maximize the uplink throughput by optimally allocating the time for the downlink wireless energy transfer by the WMV and the uplink transmissions of different sensors. We consider two scenarios where the WMV moves in a straight line and around a circle. By time discretization, the optimization problem is formulated as a $0-1$ programming problem. We obtain the upper and lower bounds of the problem by converting the original 0-1 programming problem into a linear programming problem and then obtain the optimal solution by using branch and bound algorithm. We further prove that the network throughput is independent of the WMV's velocity under certain conditions. Performance of our proposed algorithm is evaluated through extensive simulations. The results validate the correctness of our proposed theorems and demonstrate that our algorithm outperforms two baseline algorithms in achieved throughput under different settings.
\end{abstract}

Keywords: Wireless rechargeable sensor networks, RF energy harvesting mobile charging, data gathering, network throughput

This work was supported in part by National Natural Science Foundation of China under grants Nos. 61772472, 61672465, Natural Science Foundation of Zhejiang Province under grant No.LY17F020020. 


\section{Introduction}

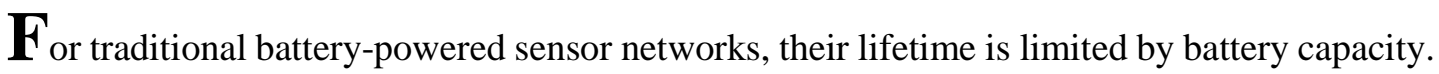
It is often difficult or impossible to replace the batteries after deployment, especially when the system is deployed in harsh and unattended environment. Although energy efficiency has been extensively studied in sensor networks [1], the problem of limited lifetime cannot be completely resolved only by saving energy [2][3]. Recent advances in radio frequency (RF) power transfer provide a promising technology to power these sensor nodes [4][5][6][7][8]. By this technology, a node can convert part of the received signal power into electricity. Depending on the transmit power of the wireless charger and the distance between the charger and the receiver, a node can harvest from some $\mu \mathrm{W}$ to some $\mathrm{mW}$ of power [9][10]. To provide stable and reliable power supply for sensor networks, adoption of mobile chargers to replenish the nodes' energy has recently attracted a lot of attention in the research community [11][12][13]. Different from traditional energy harvesting sensor networks using solar panels or wind turbines, where the harvested energy is dynamic in both spatial and temporal dimensions [12], the mobility assisted energy replenishment provides predictable and sustained power service.

In addition to harvesting environmental energy to maintain the survival of the network, how to gather sensed data is also one of the most important tasks in sensor networks [11]. There is some work investigating relay-routing based data gathering to a static data sink in energy harvesting sensor networks [13]. However, such a scheme may lead to non-uniform energy consumption among all the sensors and more congestion and packet loss at the sensors closer to the static sink. To overcome these problems, mobile data gathering has been proposed recently [11][14]. Specifically, one or more mobile sinks are employed to gather data from sensors through only one hop. This approach can effectively eliminate the non-uniformity of energy consumption among sensors and alleviate the traffic burden of sensors closer to the sink.

In this paper, we study the joint optimization of mobile charging and data gathering in sensor networks. A wireless multi-functional vehicle (WMV) is employed, which is equipped with a powerful transceiver and high capacity battery. It periodically moves along specified trajectories, charge the sensors and gather the sensed data via one-hop communication. This way, the WMV serves as both a wireless charger and a mobile data collector. Unlike prior work on SWIPT [15], which focused on the simultaneous energy and information transmissions to users in the downlink (DL), we consider a different setup where the WMV broadcasts only wireless energy to nearby sensors in the DL while the sensors transmit their independent sensed data using their individually harvested energy to the WMV in the uplink (UL). We are interested in maximizing the UL throughput by optimally allocating the time for the DL wireless energy transfer by the WMV and the UL transmissions of different sensors.

The main contributions of this paper are summarized as follows:

1) We study the joint optimization of mobile charging and data gathering problem under two scenarios that the WMV moves in a straight line and around a circle. By time discretization, the problem is formulated as a $0-1$ programming problem.

2) We first omit the integer constraint and convert the original 0-1 programming problem into a linear programming problem. Then we adopt relax-and-fix algorithm and obtain the upper bound and lower bound of the original problem. Finally, we 
obtain the optimal solution by using branch-and-bound algorithm.

3) We further study the relationship between the network throughput and the velocity of the WMV. We prove that the network throughput is independent of the WMV's velocity under certain conditions.

4) We evaluate the performance of our proposed algorithm through extensive simulations, and the results validate the correctness of our proposed theorems and demonstrate that our algorithm outperforms two baseline algorithms in achieved throughput under different settings.

The rest of this paper is organized as follows. In Section 2, we present related work. Section 3 introduces the system model. In Section 4, we formally formulate the problem under the scenario that the WMV moves in a straight line and propose algorithms to solve the problem. Section 5 extends the problem to the scenario that the WMV moves around a circle. Section 6 presents the evaluation results obtained through simulations, and we conclude the paper in Section 7.

\section{Related Work}

In recent years, the technology of RF energy harvesting has been developed rapidly. By this technology, sensor nodes can harvest power from ambient RF signals or from dedicated RF chargers. Compared to ambient RF energy [16][17] or using fixed chargers [10], it is more flexible to use mobile RF chargers. Among the studies of adopting mobile RF chargers, most are devoted to maximizing the charging efficiency. For example, Ye et al. study the problem of maximizing the charging utility by selecting the proper anchor point of the vehicle [18]. Shu et al. propose a suboptimal velocity control strategy for the mobile charger, aiming to maximize the harvested energy of nodes [19]. Fu et.al study how to plan the optimal movement strategy of the mobile charger, such that the time to charge all nodes in the network above their energy threshold is minimized [20]. Li et al optimize the trajectory of the mobile charger, aiming to reduce the moving time of the charger so that more time can be allocated to the nodes for charging and data collecting [21]. Some work focuses on solving the problem of energy imbalance among nodes. For example, Han et al. design the residence time of each layer of the transmitter through the grid model to achieve smaller variance of the node's residual energy [22]. Salarian et al. propose a path selection strategy for mobile sink to optimize the energy balance of nodes [23].

In addition to harvesting energy to prolong network lifetime, how to gather sensed data is one of the most important issues in WSNs. Among the studies of this problem, most are devoted to maximizing the network throughput. For example, Zhang et al. formulate a data gathering problem with the objective of maximizing the network utility in a rechargeable wireless sensor network with a mobile sink, where the network utility is related to the amount of sensed, transmitted and received data [11]. Mehrabi et al. optimize the time to transmit data for each node by considering two adjacent slot energy constraint to increase the network throughput [24]. According to the communication quality between each sensor and the hybrid access point, Ju and Zhang assign the charging time and the data transmission time of each node to maximize the sum-throughput and common-throughput [25]. They further reduce the energy imbalance between nodes by cooperation among nodes [26].

However, very few researches focus on joint optimization of mobile charging and data gathering. For instance, Guo et. al provide a distributed and adaptive solution that jointly selects the sensors to be recharged, finds the optimal data generating and uploading rates and 
the optimal scheduling and routing paths for each node, and determines the optimal sojourn time for the mobile collector at each anchor point, such that the overall network utility can be maximized [14]. Zhang et.al study data gathering problem in rechargeable sensor networks with a mobile sink, where rechargeable sensors are deployed into a region of interest to monitor the environment and a mobile sink travels along a pre-defined path to collect data from sensors periodically. However, the sensors in their study are powered by solar cell and rechargeable batteries, not by a RF charger [11]. Our work is different from the above work. First, we introduce a WMV which can function as both a wireless charger and a data collector. Second, we consider a relatively small sensor network, where the sensors can communicate with the WMV in one hop. Third, we assume the WMV is moving continuously along a predetermined path, a straight line or a circle, through the network without stops. In the scenario that the WMV stays at some anchor points to charge the sensors and collect data, there are mainly two limitations: first, the anchor points should be carefully chosen so as to ensure the efficiency of charging and data collection. Second, the WMV cannot charge the sensors and collect data when it is moving. Adoption of the non-stop moving model can avoid the above-mentioned two limitations. In practical applications, the WMV can be any non-dedicated vehicle in the road. They can charge the sensors at roadside and meanwhile collect the sensing data. In practical, the WMV can also move along any route. The reason for considering a straight line or a circle in our work is that most complicated routes can be approximately decomposed into the two basic motion patterns. The objective of our work is to maximize the throughput by optimally allocating the time for the wireless energy transfer by the WMV and the transmissions of different sensors.

\section{System Model}

A potential application scenario considered in this paper is given in Fig. 1. This network consists of $n$ rechargeable sensor nodes and a WMV in a 2D area. The WMV traverses the sensor network at a velocity of $v$ along a straight line, see the dotted line $A B$ in Fig. 1. Suppose the length of the segment $A B$ is $l$ and the duration of the WMV moving from $A$ to $B$ is $T$. We further make the following assumptions:

1) The WMV recharges the sensors with RF energy by broadcasting wireless signals in the DL.

2) The sensors transmit their independent sensed data using their individually harvested energy to the WMV in one hop in the UL by time-division-multiple-access (TDMA).

3) The WMV has only one antenna, so power recharge and data transmission cannot be conducted simultaneously.

4) In the whole process, each node can communicate with the WMV, and the WMV has enough energy to recharge the sensors and gather the sensed data from the sensors. 


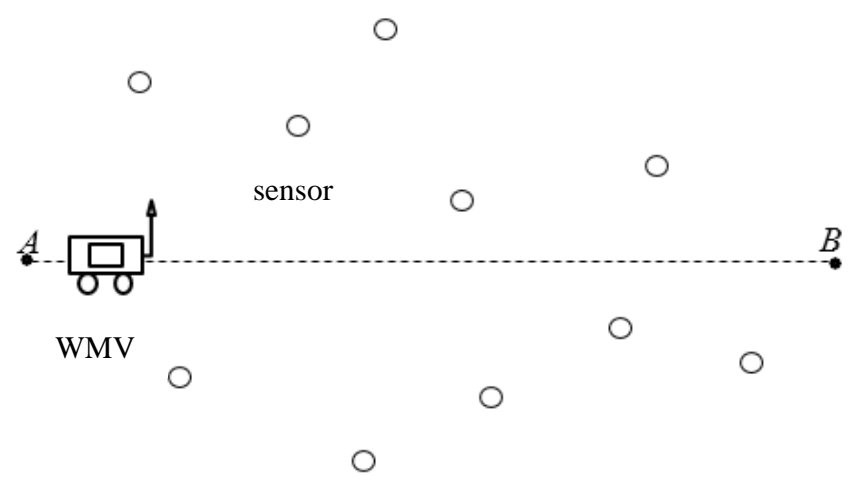

Fig. 1. The scenario that the WMV moves in a straight line to charge the sensors and gather data

When the WMV is recharging the sensors, the recharge power of the sensor is mainly related to the transmit power of the WMV and the distance between the sensor and the WMV. Since the WMV is moving continuously, the distance between the sensor and the WMV is varying. As a result, the recharge power of each sensor is dynamic. Thus, the instantaneous recharge power at time $t(0 \leq t \leq T, t=0$ represents the start of the period) of node $i$ is expressed as [25]:

$$
P^{(r)}{ }_{i}(t)=\zeta_{i} \square_{i}(t) P_{A}
$$

where $0<\zeta_{i}<1, i=1, \ldots, n$, is the energy harvesting efficiency of sensor $i, P_{A}$ denotes the transmit power of the WMV, and $h_{i}(t)=10^{-3} \varphi_{i}^{2} d_{i}^{-\alpha}(t)$ denotes the channel power gains in the DL at time $t$, where $\varphi_{i}$ represents the additional channel short-term fading which is assumed to be Rayleigh distributed, and thus $\varphi_{i}^{2}$ is an exponentially distributed random variable with unit mean, $d_{i}(t)$ is the distance between the WMV and node $i$ at time $t$, and $\alpha$ denotes the path loss exponent which is normally between 2 to 4 .

We assume that any sensor $i$ transmits data to the WMV at a constant power $P_{i}$. Then the achievable UL throughput of sensor $i$ at time $t$ can be expressed as:

$$
r_{i}(t)=B \log _{2}\left(1+\frac{g_{i}(t) P_{i}}{\Gamma \sigma^{2}}\right)
$$

where $B$ is the bandwidth, $\Gamma$ represents the signal-to-noise ratio (SNR) gap from the additive white Gaussian noise (AWGN) channel capacity due to a practical modulation and coding scheme (MCS) used, $\sigma^{2}$ denotes the variance of the noise, and $g_{i}(t)=10^{-3} \varphi_{i}^{2} d_{i}^{-\alpha}(t)$ denotes the channel power gains in the UL at time $t$.

The main notations and their corresponding definitions are summarized in Table 1.

Table 1. List of notations

\begin{tabular}{c|l}
\hline Notation & \multicolumn{1}{c}{ Definition } \\
\hline \hline$n$ & Number of sensor nodes \\
$\tau$ & Number of slots \\
$T$ & Slot duration \\
$l$ & Period duration \\
$d_{h}$ & Moving distance when the WMV moves in a straight line \\
$P_{i}$ & The radius of the circle when the WMV moves around a circle \\
$P_{i}^{(r)}$ & Transmit power of sensor $i$ \\
$r_{i}$ & Recharge power of sensor $i$
\end{tabular}




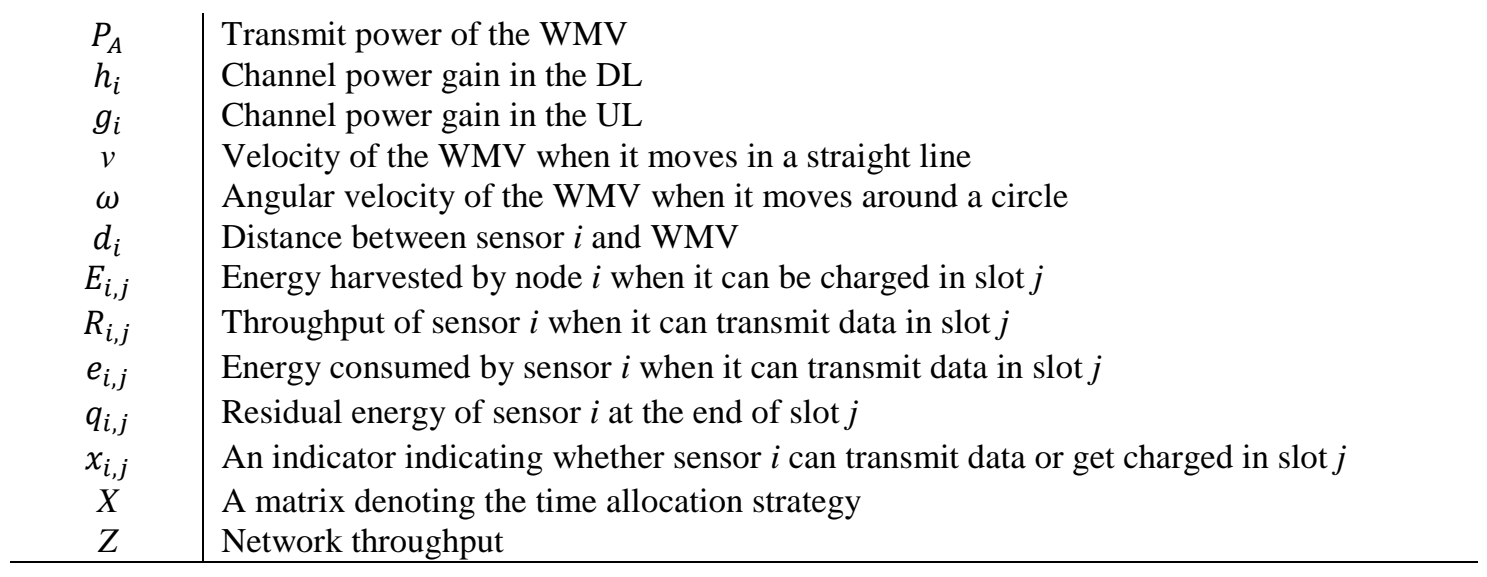

\section{Problem Formulation and Solution}

With the aforementioned models, we describe and mathematically formulate our problem in this section.

\subsection{Problem formulation}

The objective is to maximize the UL throughput by optimally allocating the time for the DL wireless energy transfer by the WMV and the UL transmissions of different sensors during the time $T$ when the WMV is moving from $A$ to $B$. We abbreviate this optimal mobile charging and data gathering problem as OMCDG.

Since the original OMCDG problem is continuous in the time domain. To make the problem easier to handle, we discretize the time duration $T$ into $m$ slots and the duration of each slot is $\tau$. So, we have $m \tau=T$. The Cartesian coordinate system is established by taking $A$ as the origin and the $A B$ direction as the positive axis of the $x$-axis. Accordingly, the coordinates of sensor $i$ are $\left(x_{i}, y_{i}\right)$. The distance between sensor $i$ and the WMV at time $t$ is given by:

$$
d_{i}(t)=\left(\left(v t-x_{i}\right)^{2}+y_{i}^{2}\right)^{\frac{1}{2}}
$$

The traversing distance of the WMV in the $j$-th slot is the accumulated distance in the previous $j$ - 1 time slots plus the incremental distance in the $j$-th slot. So, the channel power gain of DL and UL for time $\gamma(0 \leq \gamma \leq \tau, \gamma=0$ represents the start of one slot) in the $j$-th $(1 \leq j \leq m)$ slot can be expressed as:

$$
\square_{i, j}(\gamma)=g_{i, j}(\gamma)=10^{-3} \varphi_{i}^{2}\left(x_{i}^{\prime}{ }^{2}+y_{i}{ }^{2}\right)^{-\frac{\alpha}{2}}
$$

where $x^{\prime}{ }_{i}=j v \tau-v \tau+v \gamma-x_{i}$. From (1) and (4), the energy harvested by sensor $i$ when it is charging in slot $j$ is given by:

$$
E_{i, j}=\int_{0}^{\tau} \zeta_{i} P_{A} \square_{i, j}(\gamma) d \gamma
$$

Based on (2) and (4), the throughput that sensor $i$ can achieve when it transmits data in slot $j$ is given by:

$$
R_{i, j}=\int_{0}^{\tau} B \log _{2}\left(1+\frac{g_{i, j}(\gamma) P_{i}}{\Gamma \sigma^{2}}\right) d \gamma
$$

In addition, the energy consumed by sensor $i$ when it transmits data in slot $j$ is given by:

$$
e_{i, j}=P_{i} \tau
$$


Let $x_{i, j}$ be an binary indicator indicating whether sensor $i$ can transmit data in slot $j$. If it transmits data in slot $j, x_{i, j}=1$. Otherwise $x_{i, j}=0$. Specifically, $x_{0, j}$ is to indicate whether the WMV can recharge the sensors in slot $j$. If it recharges the sensors, $x_{0, j}=1$, otherwise $x_{i, j}=0$. Since power recharge and data transmission cannot be conducted simultaneously and the transmission follows TDMA, we have:

$$
\sum_{i=0}^{n} x_{i, j}=1, x_{i, j} \in\{0,1\}, j \in\{1,2, \ldots, m\}
$$

Let $q_{i, j}$ be the residual energy of node $i$ at the end of the $j$-th slot. Specifically, $q_{i, 0}$ represents the initial energy of sensor $i$ at the beginning of the period. The harvested and consumed energy of sensor $i$ in the $j$-th slot are $x_{0, j} E_{i, j}$ and $x_{i, j} e_{i, j}$ respectively. We assume the battery capacity of the sensor is large enough to avoid the case of energy overflow. So, we have:

$$
q_{i, j}=q_{i, j-1}+x_{0, j} E_{i, j}-x_{i, j} e_{i, j}
$$

We further assume that sensor $i$ cannot transmit any data when its residual energy is lower than $e_{i, j}$, which is the energy demand for the transmission in the $j$-th slot, and thus we have:

$$
x_{i, j} e_{i, j} \leq q_{i, j-1}, i \in\{1,2, \ldots, n\}, j \in\{1,2, \ldots, m\}
$$

In summary, the OMCDG problem can be formulated as:

$$
\begin{aligned}
& \max _{X} Z=\frac{1}{T} \sum_{i=1}^{n} \sum_{j=1}^{m} x_{i, j} R_{i, j} \\
& \text { s.t. } \quad \sum_{i=0}^{n} x_{i, j}=1, j \in\{1,2, \ldots m\}, \\
& \quad x_{i, j} \in\{0,1\}, i \in\{0,1, \ldots, n\}, j \in\{1,2, \ldots, m\}, \\
& \quad x_{i, j} e_{i, j} \leq q_{i, j-1}, i \in\{1,2, . . n\}, j \in\{1,2, \ldots m\},
\end{aligned}
$$

where $X=\left\{x_{0,1}, \ldots, x_{n, 1}, x_{0,2}, \ldots, x_{n, 2}, \ldots, x_{0, m}, \ldots, x_{n, m}\right\}$ is solution set of the problem including a total of $(n+1) m$ elements.

\subsection{Solution to the problem}

Obviously, the above OMCDG problem is a 0-1 programming problem, which is NP-hard. Explicit enumeration is normally impossible due to the exponentially increasing number of potential solutions. We first omit the integer constraint and convert the original 0-1 programming problem into a linear programming (LP) problem. Then we adopt relax-and-fix (R\&F) algorithm and obtain two feasible solutions corresponding to the upper bound and lower bound of the original problem. Branch and bound (B\&B) is by far the most widely used tool for solving large scale NP-hard combinatorial optimization problems. We obtain the optimal solution by using B\&B algorithm.

Specifically, for the OMCDG problem, we first relax the binary variables $x_{i, j}$ from $\{0,1\}$ to real numbers in the range $[0,1]$. The original problem is then converted into a LP problem. We obtain the solution set $\bar{X}$ of this LP problem by using CPLEX optimizer [27] and the corresponding throughput $\bar{Z}$ is the upper bound of the original problem. Then, we find the element with the minimum non-zero value in the solution set $\bar{X}=\left\{x_{0,1}, \ldots, x_{n, 1}, x_{0,2}, \ldots, x_{n, 2}, \ldots, x_{0, m}, \ldots, x_{n, m}\right\}$, and let it be 0 if it does not equal to 1 . A new LP problem is thus formulated. We iteratively solve the new LP problem and let the minimum non-zero element in respective solution set be 0 until the minimum non-zero element equals to 1. Finally, we obtain the solution set $\underline{X}$ and the corresponding throughput $\underline{Z}$ is the lower bound of the original problem. The pseudocode of R\&F algorithm for obtaining the upper and lower bounds of the OMCDG problem is shown in Table 2. 
Table 2. R\&F algorithm for obtaining the upper and lower bounds of the OMCDG problem

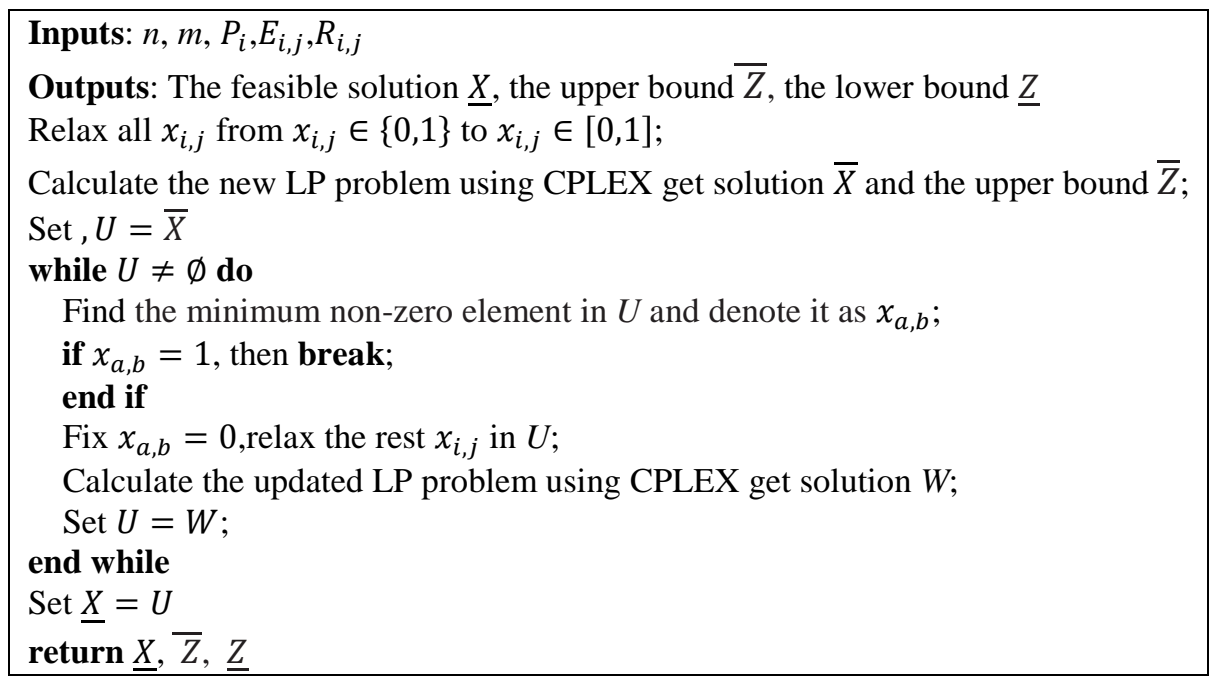

Next, we adopt B\&B algorithm to obtain the optimal solution of the OMCDG problem. In fact, for solving OMCDG problem, we need to determine whether the WMV charges the sensors $\left(x_{0, j}=1\right)$ or the sensor transmits data $\left(x_{0, j}=0\right)$, and which sensor transmits the data $\left(x_{i, j} \in\{0,1\}\right)$ in each slot. To this end, we generate $n+1$ branches for each slot when searching the solution space, where the first branch represents that WMV charges the sensors, namely, $\left\{x_{0, j}=1, x_{i, j}=0, i \neq 0\right\}$ and any other branch represents that any sensor $i$ transmits data, namely, $\left\{x_{i, j}=1, x_{k, j}=0, k \neq i\right\}$. Since we have the upper and lower bounds obtained by $\mathrm{R} \& \mathrm{~F}$ algorithm, we can reduce the searching space by cutting some of the branches and updating the upper and lower bounds during the searching process. The details of B\&B algorithm for solving the OMCDG problem are as follows: In the first slot, we generate $n+1$ branches corresponding to the solutions $\left\{x_{0,1}=1, x_{i, 1}=0, i \neq 0\right\},\left\{x_{1,1}=1, x_{i, j}=0, i \neq\right.$ $1\},\left\{x_{2,1}=1, x_{i, j}=0, i \neq 2\right\}, \ldots,\left\{x_{n, 1}=1, x_{i, j}=0, i \neq n\right\}$. We then solve $n+1$ LP problems with fixed solution in the first slot and relaxed variables $x_{i, j}(j>1)$ in the remaining slots, and obtain $n+1$ solutions and corresponding throughputs. We find the solution $X_{\max }$ that has maximum throughput, denoted by $Z_{\max }$ and let $\bar{Z}=Z_{\max }$. After that, we check whether there exist solutions conforming to the integer constraint. If true, we denote the solution that has maximum throughput by $X_{\max }^{\prime}$ and the throughput by $Z_{\max }^{\prime}$ and further compare $Z_{\max }^{\prime}$ with current lower bound $\underline{Z}$. If $Z_{\max }^{\prime}>\underline{Z}$, let $\underline{Z}=Z_{\max }^{\prime}, \underline{X}=X_{\max }^{\prime}$. Otherwise, we cut off the branches which are unsolvable or whose throughput is no more than current lower bound $\underline{Z}$ and enter the iteration of the second slot. The iteration terminates when all the slots have been visited or $\bar{Z}-\underline{Z} \leq \epsilon$, where $\epsilon$ is a small controllable error coefficient. Finally, we obtain the optimal solution $X^{*}=\underline{X}$ and its corresponding throughput $\underline{Z}$. The pseudocode of B\&B algorithm for obtaining the optimal solution of the OMCDG problem is shown in Table 3. 
Table 3. B\&B algorithm for obtaining the optimal solution of the OMCDG problem

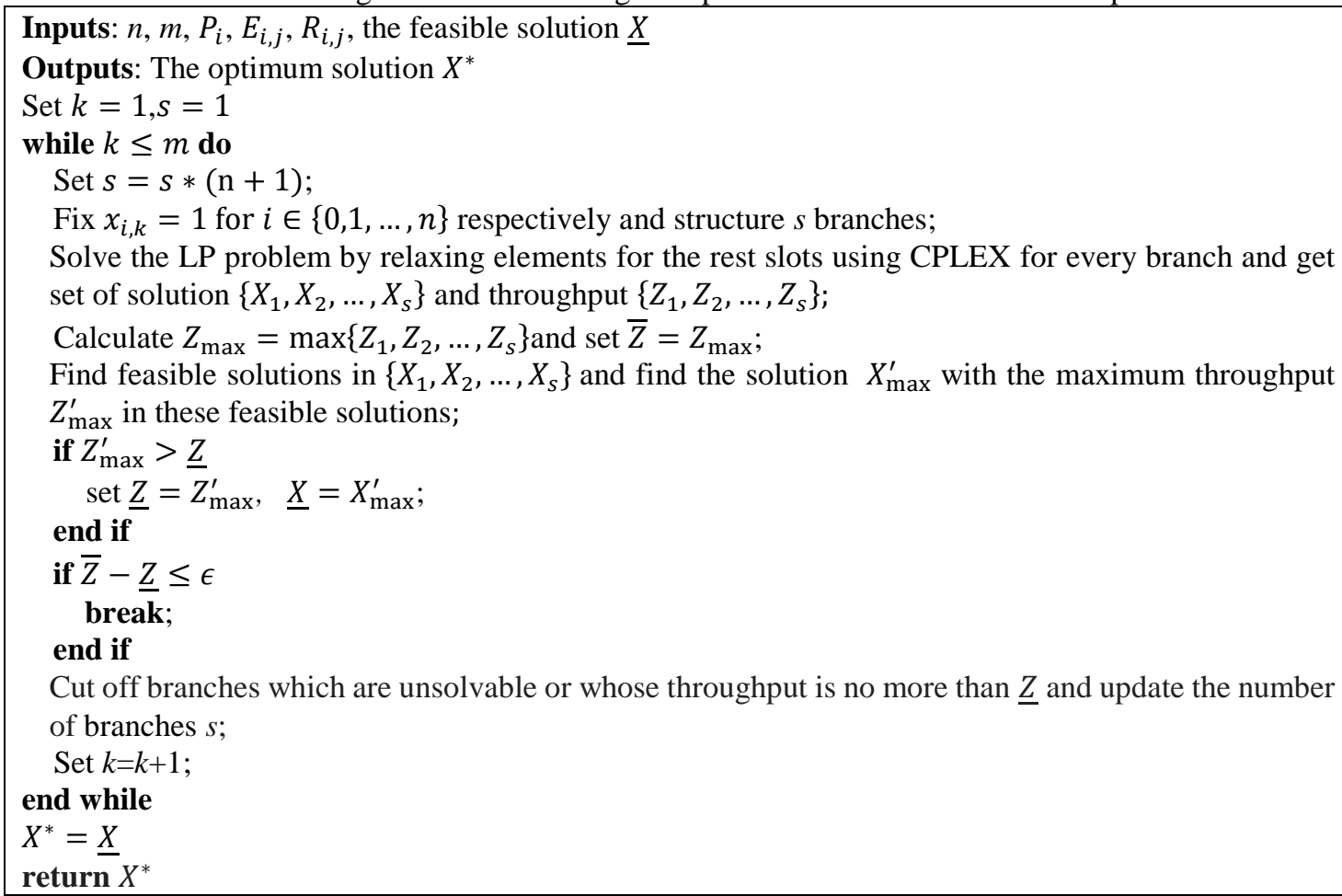

Complexity analysis: Both the number of variables and the number of constraints of the LP problem are $(m n+m)$. It is known that the time complexity of solving LP problems by using interior point method is $\mathrm{O}(m n+m)^{3.5}$ [28]. Since we need $(m n+m)$ loops to obtain the feasible solution $\underline{X}$ under the worst case and the complexity for each loop is $\mathrm{O}((\mathrm{mn}+$ $\left.m)(m n+m)^{3.5}\right)$, the complexity of R\&F algorithm is $\mathrm{O}\left((m n+n)^{5.5}\right)$. Under the worst case, we need $(n+1)^{m}$ loops to obtain the optimal solution $X^{*}$. Obviously, when the number of sensors or slots is relatively large, the cost is unaffordable. Since we introduce a small and controllable error coefficient $\epsilon$, we can jump out of the iteration if $\bar{Z}-\underline{Z} \leq \epsilon$, which results in an approximate solution, but can greatly reduce the time complexity.

\subsection{Further discussions}

In this section, we further discuss the relationship between the network throughput and the velocity of the WMV by proposing and proving three theorems.

Theorem 1. If the WMV moves in a straight line with a constant velocity and broadcasts power signals continuously, the average harvested power of the sensors is independent of the velocity of the WMV given a fixed moving path.

Proof. Since the WMV broadcasts power signals continuously, sensor $i$ can harvest energy all the time when the WMV moves from $A$ to $B$. Let $v$ be the velocity of the WMV, $l$ be the length of the line segment $A B$, and $\left(x_{i}, y_{i}\right)$ be the coordinates of sensor $i$. From (1) and (3), the total harvested energy $E_{i}$ by sensor $i$ can be expressed as

$$
E_{i}=\int_{0}^{T} 10^{-3} \zeta_{i} \varphi_{i}^{2} P_{A}\left(\left(v t-x_{i}\right)^{2}+y_{i}^{2}\right)^{-\frac{\alpha}{2}} d t
$$


Let $\lambda_{i}=10^{-3} \zeta_{i} \varphi_{i}^{2} P_{A}$, then the average recharge power is

$$
P^{(r)}{ }_{i}=\frac{E_{i}}{T}=\lambda_{i} T^{-1} \int_{0}^{T}\left(\left(v t-x_{i}\right)^{2}+y_{i}^{2}\right)^{-\frac{\alpha}{2}} d t
$$

We further let $\delta=v t$, this way $d \delta=v d t$, and thus (13) is rewritten as:

$$
P^{(r)}{ }_{i}=\lambda_{i}(v T)^{-1} \int_{0}^{v T}\left(\left(\delta-x_{i}\right)^{2}+y_{i}^{2}\right)^{-\frac{\alpha}{2}} d \delta
$$

Let $f(\delta)=\left(\left(\delta-x_{i}\right)^{2}+y_{i}^{2}\right)^{-\alpha / 2}$, and obviously $f(\delta)$ is continuous in the range [0,vT]. According to the mean value theorems for integrals, there exists a $\varepsilon \in[0, v T]$ that satisfies:

$$
P^{(r)}{ }_{i}=\lambda_{i}(v T)^{-1} f(\varepsilon)(v T-0)=\lambda_{i} f(\varepsilon)
$$

Since $v T=l$ is the length of this path, the integral interval $[0, v T]$ is the same for different $v$. As a result, $\varepsilon$ and $f(\varepsilon)$ are also the same. In other words, $f(\varepsilon)$ is independent of $v$. From (15), we prove that the average recharge power of sensor $i$ harvesting from the WMV is independent of the velocity $v$. The theorem follows immediately.

Theorem 2. Suppose the sensor has sufficient energy and transmits data with a constant transmit power to the WMV moving in a straight line with a constant velocity, the throughput of the sensor is independent of the velocity of the WMV given a fixed moving path.

Proof. Let $g_{i}(t)=10^{-3} \varphi_{i}^{2}\left(\left(v t-x_{i}\right)^{2}+y_{i}^{2}\right)^{-\frac{\alpha}{2}}$, and suppose the transmit power of sensor $i$ is $P_{i}$, from (2) and (3), the throughput of sensor $i$ can be expressed as:

$$
r_{i}=B T^{-1} \int_{0}^{T} \log _{2}\left(1+\frac{g_{i}(t) P_{i}}{\Gamma \sigma^{2}}\right) d t
$$

Similar to the proof of theorem 1 , the result can be obtained by using the mean value theorems for integrals. Let $\delta=v t, f^{\prime}(\delta)=\log _{2}\left(1+\frac{g_{i}(\delta) P_{i}}{\Gamma \sigma^{2}}\right)$, we can easily prove that there exists a $\varepsilon$, $\in[0, v T]$ that satisfies $r_{i}=B f^{\prime}\left(\varepsilon^{\prime}\right)$ and thus $f^{\prime}\left(\varepsilon^{\prime}\right)$ is independent of the velocity $v$ of the WMV. The theorem follows immediately.

Theorem 3. If all the sensors have no initial power and the WMV traverses the sensor network at a constant velocity, following the schedule obtained by $B \& B$ algorithm to charge the sensors and gather the sensed data, the throughput of the whole network is independent of the moving velocity.

Proof. Suppose there are two velocities $v_{1}$ and $v_{2}$, the velocity $v_{1}$ is $\beta$ ( $\beta$ is a positive number and $\beta \neq 1$ ) times of $v_{2}$, i.e. $v_{1}=\beta v_{2}$. Then we have:

$$
\begin{aligned}
& T_{2}=\beta T_{1} \\
& \tau_{2}=\beta \tau_{1}
\end{aligned}
$$

where $T_{1}$ and $T_{2}$ are the lengths of the periods, $\tau_{1}$ and $\tau_{2}$ are the length of the time slots under the two velocity settings, respectively. Similarly, let $E_{i, j}^{(1)}, E_{i, j}^{(2)}, R_{i, j}^{(1)}, R_{i, j}^{(2)}, e_{i, j}^{(1)}, e_{i, j}^{(2)}$ denote corresponding $E_{i, j}, R_{i, j}$ and $e_{i, j}$ under the two velocity settings, respectively. Let $P_{i, j}^{(r)}$ and $r_{i, j}$ be the average recharge power and the average throughput of node $i$ in slot $j$, respectively. From theorem 1 and 2, we know that $P_{i, j}^{(r)}$ and $r_{i, j}$ are the same under two velocities. Then from (18), we have:

$$
\begin{gathered}
E_{i, j}^{(2)}=P_{i, j}^{(r)} \tau_{2}=\beta E_{i, j}^{(1)} \\
R_{i, j}^{(2)}=r_{i, j} \tau_{2}=\beta R_{i, j}^{(1)} \\
e_{i, j}^{(2)}=P_{i} \tau_{2}=\beta e_{i, j}^{(1)}
\end{gathered}
$$

From (17) and (20), it is obvious that the objective functions are equivalent under the two 
velocity settings. Then we check the constraints. we only have to check constraint (11) since constraints (8) and (9) are irrelevant of the velocity. For any $i \in\{0,1, \ldots, n\}$, constraint (11) can be transformed into:

$$
\left\{\begin{array}{c}
x_{i, j} e_{i, j}-\sum_{k=1}^{j-1} \Delta E_{i, k} \leq e_{i, 0}, j \in\{2,3, \ldots, m\} \\
x_{i, j} e_{i, j} \leq e_{i, 0}, j=1
\end{array}\right.
$$

where $\Delta E_{i, k}=x_{0, k} E_{i, k}-x_{i, k} e_{i, k}$ denotes the change of the energy of sensor $i$ in slot $k$. Since $e_{i, 0}=0$, combining (22) with (19) and (21), it is easy to find that the constraint described by (22) is identical under the two velocity settings. In summary, the objective function and the constraints are exactly the same under the velocities $v_{1}$ and $v_{2}$. Therefore, the optimal solutions are also identical and we finally obtain equivalent throughput of the network under both settings. The theorem follows immediately.

Here we further briefly discuss how the moving velocity impacts on the data gathering delay. If the transmission delay is dominant in the data gathering delay, the delay is also independent of the WMV's moving velocity, because the transmission delay is inversely proportional to the throughput. But if other delays are large, like the queueing delay at the sensors and the data processing delay at the WMV, the data gathering delay is relevant to WMV's moving velocity.

\section{Extension of the Problem}

In this section, we extend the moving trajectory of the WMV from a straight line to a circle. As shown in Fig. 2, we consider a scenario where sensor nodes are distributed in a circle area with the center $A$ and the radius $d$. The WMV moves counterclockwise around the circle with the center $A$ and the radius $d_{h}$ (the dotted line in Fig. 2) with a fixed angular velocity $\omega$. The WMV charges the sensors and gathers sensed data. Similarly, we will solve the OMCDG problem under this model.

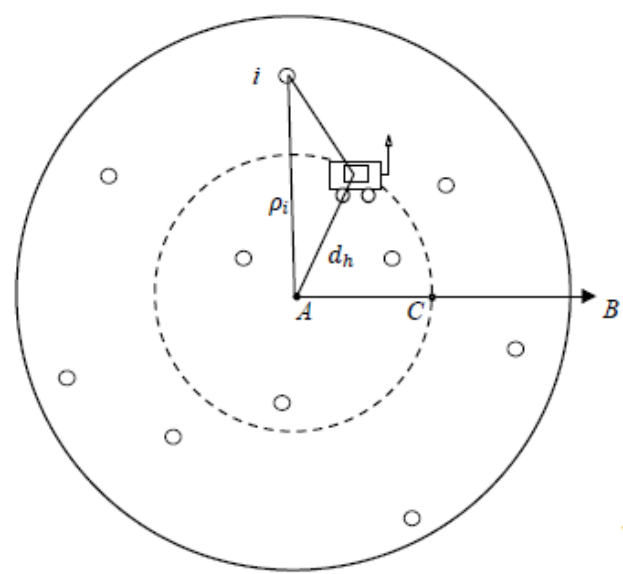

Fig. 2. The scenario that the WMV moves around a circle to charge the sensors and gather data

We take $A$ as the origin and fix a ray out from the origin in the direction of $A B$, and establish the polar coordinates. The intersection between $A B$ and the trajectory of the WMV is $C$ and the polar coordinates of sensor $i$ are $\left(\rho_{i}, \theta_{i}\right)$. Let $T$ be the duration that the WMV starts from the point $C$ and moves counterclockwise back to $C$. We study the OMCDG problem in the period of $T$. Similarly, we divide the whole period into $m$ slots and the duration of each slot is $\tau$. Let $h_{i}^{\prime}(t)$ and $g_{i}^{\prime}(t)$ be the power gains of DL and UL, respectively, and $d_{i}^{\prime}(\mathrm{t})$ be the 
distance between sensor $i$ and the WMV at time $t$. Let $E_{i, j}^{\prime}, e_{i, j}^{\prime}$ and $R_{i, j}^{\prime}$ be the harvested energy, consumed energy and throughput of sensor $i$ in slot $j$, respectively. The OMCDG problem under the circular motion model can be formulated as:

$$
\begin{array}{ll}
\max _{X} & Z=T^{-1} \sum_{i=1}^{n} \sum_{j=1}^{m} x_{i, j} R_{i, j}^{\prime} \\
\text { s.t. } & \sum_{i=0}^{n} x_{i, j}=1, j \in\{1,2, \ldots m\} \\
& x_{i, j} e_{i, j}^{\prime} \leq q_{i, j-1}, i \in\{1,2, . . n\}, j \in\{1,2, . . m\} \\
& x_{i, j} \in\{0,1\}, i \in\{0,1, \ldots, n\}, j \in\{1,2, \ldots, m\}
\end{array}
$$

We can solve the above problem using similar algorithms described in Section 4.2. The difference is the calculation of the distance between sensors and the WMV due to different motion patterns. This will further lead to difference of the harvested energy and the throughput of the sensors in each slot.

As shown in Fig. 2, in the movement of the WMV, the origin A, sensor $i$ and the WMV can always form a triangle except when the three points are on the same line. Noting that the distance between the WMV and the origin $A$ is a constant $d_{h}$ and the distance between sensor $i$ and $A$ is also a constant $\rho_{i}$. Therefore, we can calculate the distance between the WMV and node $i$ at time $t$ according to the cosine theorem. We consider the following 4 cases as shown in Fig. 3:

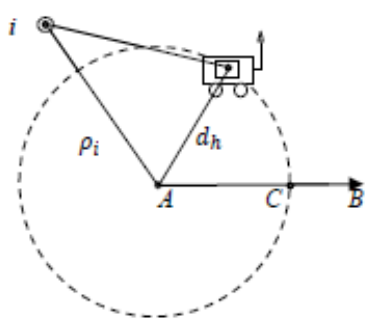

(a) case 1

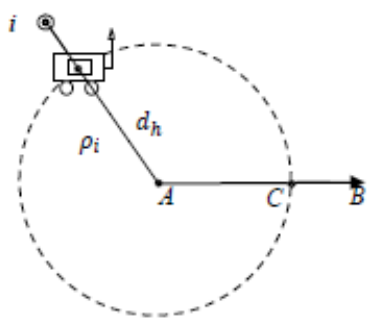

(c) case 3

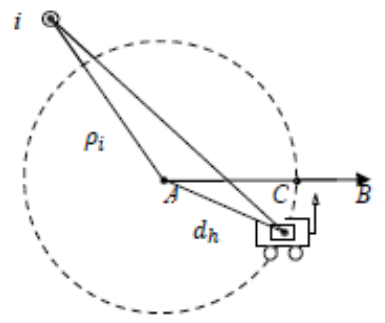

(b) case 2

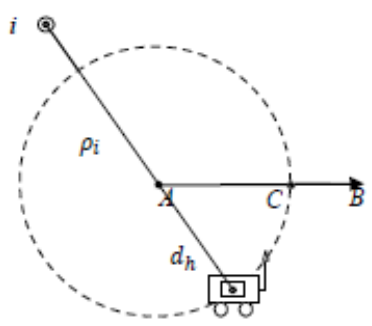

(d) case 4

Fig. 3. Four cases when the WMV moves around a circle

1) Case 1: When $\left|\theta_{i}-\omega t\right|<\pi$, as shown in Fig. 3(a), the angle between sensor $i$, origin $A$ and the WMV is $\left|\theta_{i}-\omega t\right|$. Then we have $d_{i}^{\prime}(\mathrm{t})=\left(\rho_{i}^{2}+d_{h}^{2}-2 \rho_{i} d_{h} \cos \left|\theta_{i}-\omega t\right|\right)^{\frac{1}{2}}$.

2) Case 2: When $\left|\theta_{i}-\omega t\right|>\pi$, as shown in Fig. 3(b), the angle between sensor $i$, origin $A$ and the WMV is $2 \pi-\left|\theta_{i}-\omega t\right|$. Then we have $d_{i}^{\prime}(\mathrm{t})=\left(\rho_{i}^{2}+d_{h}^{2}-2 \rho_{i} d_{h} \cos (2 \pi-\right.$ $\left.\left.\left|\theta_{i}-\omega t\right|\right)\right)^{\frac{1}{2}}$. 
3) Case 3: When $\left|\theta_{i}-\omega t\right|=0$, as shown in Fig. 3(c), since $d_{i}^{\prime}(\mathrm{t})=\left|\rho_{i}-d_{h}\right|$ and $\cos \left|\theta_{i}-\omega t\right|=1$, we have $d_{i}^{\prime}(\mathrm{t})=\left(\rho_{i}^{2}+d_{h}^{2}-2 \rho_{i} d_{h} \cos \left|\theta_{i}-\omega t\right|\right)^{\frac{1}{2}}$.

4) Case 4: When $\left|\theta_{i}-\omega t\right|=\pi$, as shown in Fig. 3(d), since $d_{i}^{\prime}(\mathrm{t})=\rho_{i}+d_{h}$ and $\cos \mid \theta_{i}-$ $\omega t \mid=-1$, we have $d_{i}^{\prime}(\mathrm{t})=\left(\rho_{i}^{2}+d_{h}^{2}-2 \rho_{i} d_{h} \cos \left|\theta_{i}-\omega t\right|\right)^{\frac{1}{2}}$.

To summarize, since cosine function is an even function, the distance between sensor $i$ and the WMV at time $t$ is:

$$
d_{i}^{\prime}(t)=\left(\rho_{i}^{2}+d_{\square}^{2}-2 \rho_{i} d_{\square} \cos \left(\theta_{i}-\omega t\right)\right)^{\frac{1}{2}}
$$

Further, the channel power gain of DL and UL at time $\gamma$ in the $j$-th $(1 \leq j \leq m)$ slot can be expressed as:

$$
h_{i, j}^{\prime}(\gamma)=g_{i, j}^{\prime}(\gamma)=10^{-3} \varphi_{i}^{2}\left(\rho_{i}^{2}+d_{\square}^{2}-2 \rho_{i} d_{\square} \cos \theta_{i}^{\prime}\right)^{-\frac{\alpha}{2}}
$$

where $\theta_{i}^{\prime}=\theta_{i}-\omega \gamma-(j \omega \tau-\omega \tau)$. After obtaining the channel power gain, we can calculate the harvested energy and the throughput of sensor $i$ following (5) and (6). Then we can obtain the feasible solutions by R\&F algorithm and further obtain the optimal solution by B\&B algorithm. Similarly, we have the following three theorems. Since the proof is very similar to that of Theorem 1, 2 and 3, we omit the details of the proof here to avoid duplication.

Theorem 4. If the WMV moves around a circle and broadcasts power signals continuously, the average harvested power of the sensors is independent of the velocity of the WMV given a fixed moving path.

Theorem 5. Suppose the sensor has sufficient energy and transmits data with a constant transmit power to the WMV moving around a circle with a constant velocity, the throughput of the sensor is independent of the velocity of the WMV given a fixed moving path.

Theorem 6. If all the sensors have no initial power and the WMV moves around a circle crossing the sensor network at a constant angular velocity, following the schedule obtained by $\mathrm{B} \& \mathrm{~B}$ algorithm to charge the sensors and gather the sensed data, the throughput of the whole network is independent of the moving velocity.

\section{Performance Evaluation}

In this section, we validate the proposed theorems and evaluate the performance of our proposed algorithm under different settings. We also implement two baseline algorithms for comparison.

\subsection{Simulation settings and baseline algorithms}

We assume that each sensor has the same transmit power, i.e. $P_{1}=P_{2}=\cdots=P_{n}=P=$ $-20 \mathrm{dBm}, \zeta_{1}=\cdots \zeta_{n}=\zeta=0.5, B=1 \mathrm{MHz}$ and $\alpha=2$. For the scenario that the WMV moves along a straight line, the velocity $v=1 \mathrm{~m} / \mathrm{s}$, and for the circle scenario, the angular velocity $\omega=\pi / 6$. The received power spectral density of the AWGN at the WMV is set to $-160 \mathrm{dBm} / \mathrm{Hz}$. Suppose an uncoded quadrature amplitude modulation (QAM) is employed and we set $\Gamma=9.8 \mathrm{~dB}$ following [25]. Other parameter settings are specified in the following section since different simulations adopt different settings.

For performance comparison, we adopt the following two baseline algorithms:

Maximum energy first (MEF) algorithm: The sensor with the maximum remaining energy will transmit data in current slot. If no sensor has enough energy in current slot, the WMV charges the sensors. The time complexity of MEF algorithm is $\mathrm{O}(m \log n)$. 
Fixed rotation (FR) algorithm: Each sensor takes turns to transmit data in each slot and the WMV charges the sensor if it does not have enough energy in its turn. The time complexity of FR algorithm is $\mathrm{O}(m)$.

\subsection{Simulation results}

In the first simulation, we consider the scenario where the WMV moves in a straight line. Suppose there are 4 sensor nodes denoted by $S_{1}, S_{2}, S_{3}$ and $S_{4}$ whose coordinates are (2, 2), (7, $-4),(14,-18)$ and $(19,3)$ respectively. Let $l=20 \mathrm{~m}, m=20$ and $P_{A}=30 \mathrm{dBm}$. First, we try to validate Theorem 3 by checking the impact of the WMV's velocity on the network throughput using our proposed B\&B algorithm, supposing that all sensors have no initial energy. As shown in Fig. 4, the network throughput remains unchanged with the increase in the WMV's velocity, which validate the correctness of Theorem 3 . Then, we try to validate Theorem 2 by checking the impact of the WMV's velocity on the throughput of each sensor, supposing that each sensor has enough energy and transmits sensed data continuously when the WMV moves from $A$ to $B$. The simulation results are shown in Fig. 4. We can observe that the throughput of each sensor also remains unchanged although the velocity of the WMV is increasing. This result has validated the correctness of Theorem 2 .

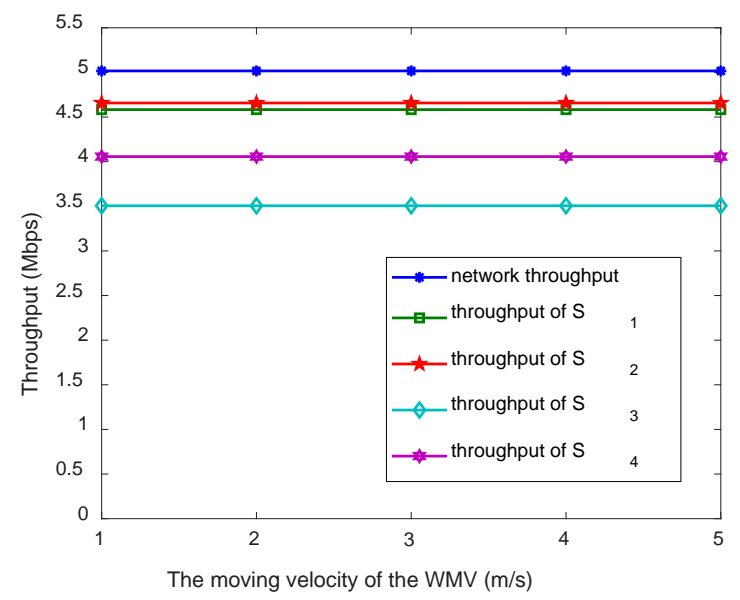

Fig. 4. The throughput vs. the velocity of the WMV when it moves along a straight line

In the second simulation, we consider the scenario where the WMV moves around a circle. Suppose there are 4 sensor nodes denoted by $S_{1}, S_{2}, S_{3}$ and $S_{4}$ whose polar coordinates are $(2, \pi / 6),(12, \pi),(7,3 \pi / 2)$ and $(15,7 \pi / 4)$ respectively. Let $d_{h}=8 \mathrm{~m}, m=20$ and $P_{A}=$ $30 \mathrm{dBm}$. Similarly, we try to validate Theorem 5 and Theorem 6 by checking the impact of the WMV's angular velocity on the network throughput and the throughput of each sensor under different conditions. The simulation results are shown in Fig. 5 and we can observe that the network throughput and the throughput of each sensor is independent of the angular velocity, which have validated the correctness of the two theorems. 


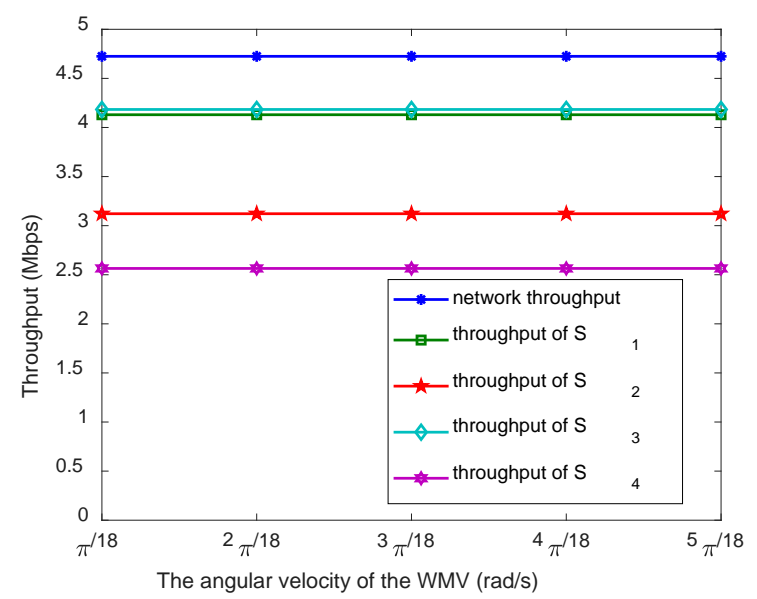

Fig. 5. The throughput vs. the angular velocity of the WMV when it moves around a circle

In the following simulations, we compare the average network throughput performances obtained by our proposed B\&B algorithm and the baseline algorithms under two scenarios where the WMV moves in a straight line and around a circle. In the scenario where the WMV moves in a straight line, sensors are randomly distributed in a square region with the coordinates satisfy $\mathrm{x}_{i} \in[0,20], \mathrm{y}_{i} \in[-10,10]$, while in the scenario where the WMV moves around a circle, sensors are randomly distributed in a circular region with the polar coordinates satisfying $\rho_{i} \in[0,16], \theta_{i} \in[0,2 \pi]$. Each result in the following simulation diagrams stands for the average value of 100 instances with different random sensor locations.

Firstly, we study the impact of the transmit power of the WMV on the network throughput. The simulation results under two different moving scenarios are shown in Fig. 6 and Fig. 7 respectively. The curves in both figures present similar tendency. As observed, the network throughput obtained by all the three algorithms increases with the increase in the WMV's transmit power. However, the improvement is decreasing and the throughput finally converges to a fixed value. This is because when the transmit power of the WMV is small, sensors take more time to get charged before transmission. As the transmit power of the WMV increases, sensors take less time to get charged and more time for transmission. But when all sensors have enough energy for transmission, the throughput cannot be further improved since the transmit power of each sensor is fixed. In general, our proposed B\&B algorithm outperforms the baseline algorithms FR and MEF. Specifically, in Fig. 6, the performance gain of B\&B algorithm over MEF algorithm is $214.67 \%$ at most and $85.39 \%$ on average, while its performance gain over FR algorithm is $29.07 \%$ at most and $21.00 \%$ on average. In Fig. 7, The performance gain of B\&B over MEF is $127.77 \%$ at most and $80.51 \%$ on average, while its performance gain over FR is $13.14 \%$ at most and $9.83 \%$ on average. 


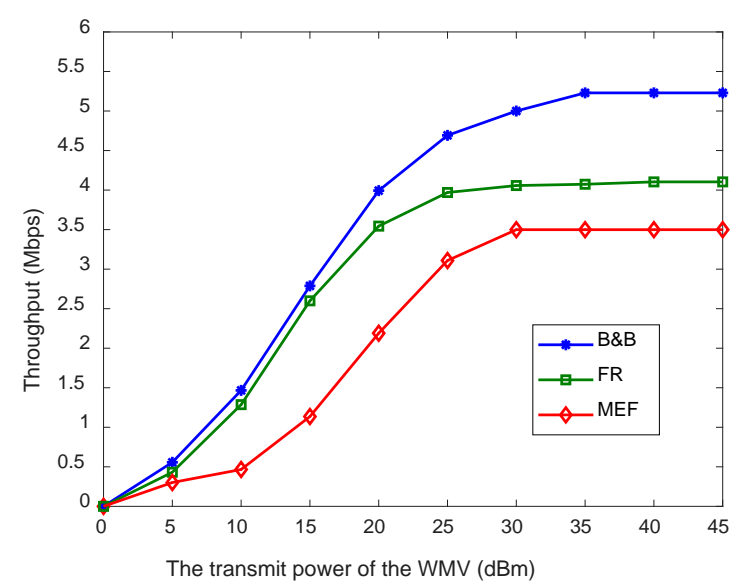

Fig. 6. The network throughput obtained by three algorithms vs. the transmit power of the WMV when it moves along a straight line, $m=20, n=4$

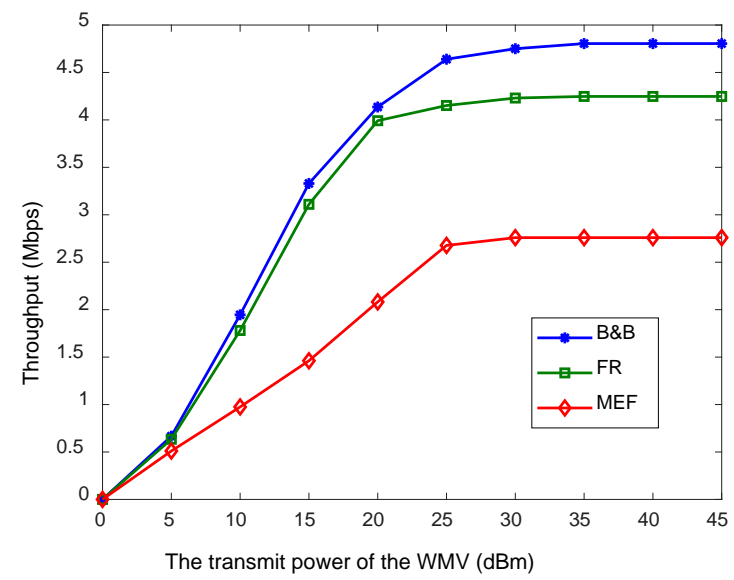

Fig. 7. The network throughput obtained by three algorithms vs. the transmit power of the WMV when it moves around a circle, $m=24, n=4$

Secondly, we study the impact of the number of slots on the network throughput. The simulation results under two different moving scenarios are shown in Fig. 8 and Fig. 9 respectively. The curves in both figures present similar tendency. As we can see, the throughput obtained by all the three algorithms does not consistently increase with the increases in the number of time slots. Suppose the number of slots is $x$, we can only ensure that the throughput with the slot number setting to $k x(k>1, k \in Z)$ is larger than that with the slot number setting to $x$. As the number of slots is large enough, the three algorithms have little change in the throughput. This is because as the number of slots is getting larger, the slot duration is getting smaller, which approximates the situation under continuous time. In general, our proposed B\&B algorithm outperforms the baseline algorithms FR and MEF. Specifically, in Fig. 8, the performance gain of B\&B algorithm over MEF algorithm is $77.04 \%$ at most and $66.64 \%$ on average, while its performance gain over FR algorithm is $32.63 \%$ at most and $28.85 \%$ on average. In Fig. 9, The performance gain of B\&B over MEF is $68.80 \%$ at most and $64.80 \%$ on average, while its performance gain over FR is $31.76 \%$ at most and $27.23 \%$ on average. 


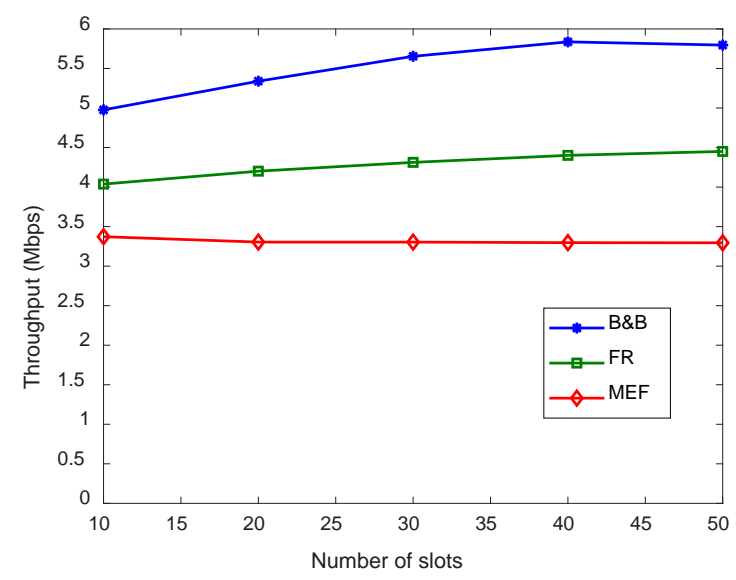

Fig. 8. The network throughput obtained by three algorithms vs. the number of slots when the WMV moves along a straight line, $P_{A}=30 \mathrm{dBm}, n=4$

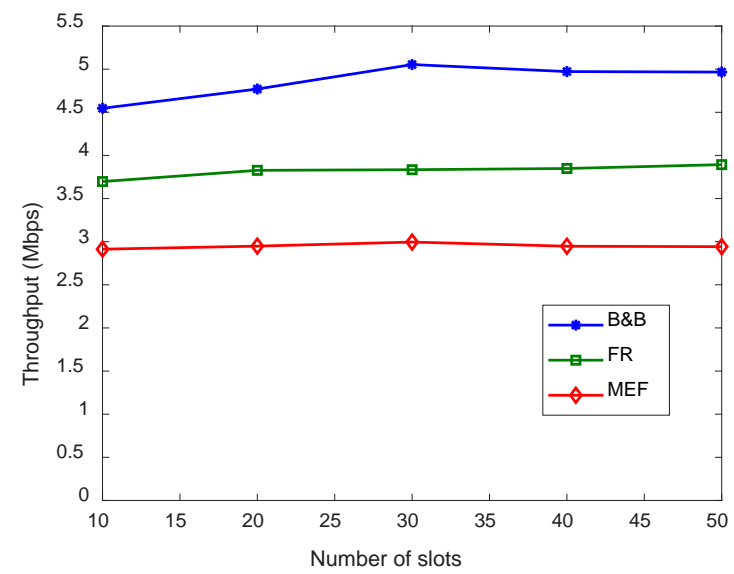

Fig. 9. The network throughput obtained by three algorithms vs. the number of slots when the WMV moves around a circle, $P_{A}=30 \mathrm{dBm}, n=4$

Finally, we study the impact of the number of sensors on the network throughput. The simulation results under two different moving scenarios are shown in Fig. 10 and Fig. 11 respectively. The curves in both figures present similar tendency. As we can observe, when the number of sensors increases, the network throughput obtained by all the three algorithms almost increases linearly. This is because the WMV can charge multiple sensors simultaneously. So, when the number of sensors increases, more sensors can contribute to the network throughput. As expected, our proposed B\&B algorithm outperforms the baseline algorithms FR and MEF. Specifically, in Fig. 10, the performance gain of B\&B algorithm over MEF algorithm is $48.06 \%$ at most and $43.39 \%$ on average, while its performance gain over FR algorithm is $25.59 \%$ at most and $18.93 \%$ on average. In Fig. 11, The performance gain of B\&B over MEF is $72.55 \%$ at most and $58.43 \%$ on average, while its performance gain over FR is $30.82 \%$ at most and $22.58 \%$ on average. 


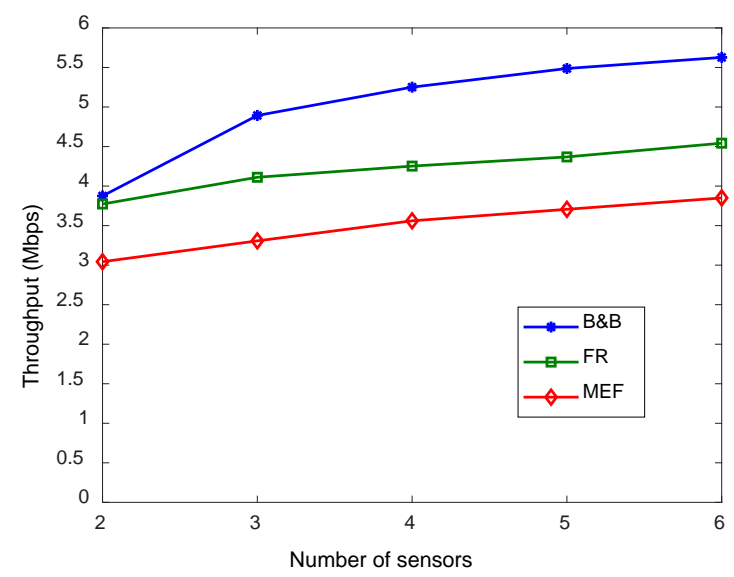

Fig. 10. The network throughput obtained by three algorithms vs. the number of sensors when the WMV moves along a straight line, $P_{A}=30 \mathrm{dBm}, m=20$

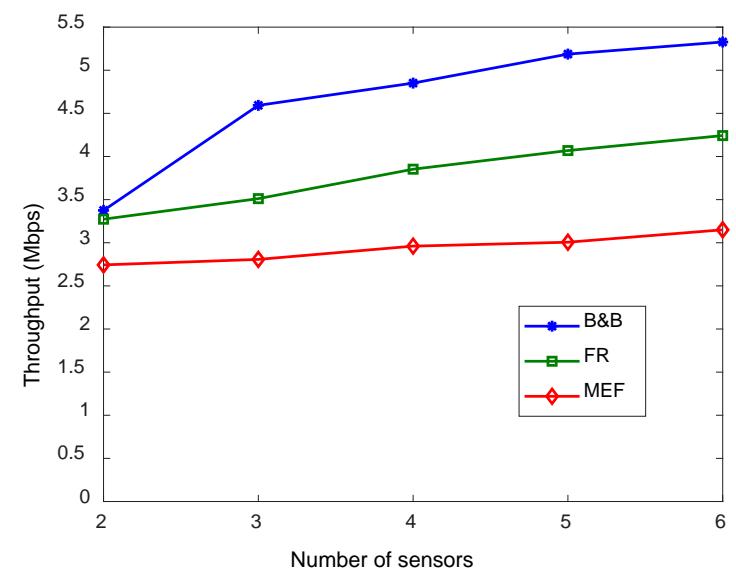

Fig. 11. The network throughput obtained by three algorithms vs. the number of slots when the WMV moves around a circle, $P_{A}=30 \mathrm{dBm}, m=24$

\section{Conclusion}

In this paper, we study the joint optimization of mobile charging and data gathering in sensor networks, with the objective of maximizing the uplink throughput by optimally allocating the time for the downlink wireless energy transfer by the WMV and the uplink transmissions of different sensors. We consider two scenarios where the WMV moves in a straight line and around a circle to charge sensors and gather data. By time discretization, the optimization problem is formulated as a 0-1 programming problem. We obtain the upper and lower bounds of the problem by converting the original 0-1 programming problem into a linear programming problem and then obtain the optimal solution by using branch and bound algorithm. We further prove that the network throughput is independent of the WMV's velocity under certain conditions. Performance of our proposed algorithm is evaluated through extensive simulations. The results validate the correctness of our proposed theorems and demonstrate that our algorithm outperforms two baseline algorithms in achieved throughput under different settings. 


\section{References}

[1] T. Rault, A. Bouabdallah, Y. Challal, "Energy efficiency in wireless sensor networks: A top-down survey,” Computer Networks, vol. 67, pp. 104-122, July, 2014. Article (CrossRef Link).

[2] Y. Li, J. Pan, and X. Tian, “A utility-based and QoS-aware power control scheme for wireless body area networks,” KSII Transactions on Internet \& Information Systems, vol. 10, no. 9, pp. 4188-4206, September, 2016. Article (CrossRef Link).

[3] H. Zhang, Y. Qi, J. Wu, L. Fu, and L. He, "DoS attack energy management against remote state estimation,” IEEE Transactions on Control of Network Systems, vol. 5, no. 1, pp. 383-394, March, 2018. Article (CrossRef Link).

[4] X. Lu, P. Wang, D. Niyato, D. Kim and Z. Han, "Wireless networks with RF energy harvesting: A contemporary survey,” IEEE Communications Surveys and Tutorials, vol. 17, no. 2, pp. 757-789, Secondquarter, 2015. Article (CrossRef Link).

[5] D. Niyato, D. I. Kim, M. Maso, and Z. Han, "Wireless powered communication networks: Research directions and technological approaches," IEEE Wireless Communications, vol. 24, no. 6, pp. 88-97, December, 2017. Article (CrossRef Link).

[6] H. Dai, Y. Liu, G. Chen, X. Wu, T. He, A. X. Liu and Y. Zhao, "SCAPE: safe charging with adjustable power,” IEEE/ACM Transactions on Networking, vol. 26, no. 1, pp.520-533, February, 2018. Article (CrossRef Link).

[7] H. Dai, H. Ma, A.X. Liu and G. Chen, "Radiation constrained scheduling of wireless charging tasks,” IEEE/ACM Transactions on Networking, vol. 26, no. 1, pp. 314-327, February, 2018. Article (CrossRef Link).

[8] H. Zhang and W.X. Zheng, "Robust transmission power management for remote state estimation with wireless energy harvesting,” IEEE Internet of Things Journal, vol. 5, no. 4, pp. 2682-2690, August, 2018. Article (CrossRef Link).

[9] Y. Li, L. Fu, Y. Ying, Y. Sun, K. Chi, and Y. Zhu, "Goodput optimization via dynamic frame length and charging time adaptation for backscatter communication," Peer-to-Peer Networking and Applications, vol. 10, no. 3, pp. 440-452, May, 2017. Article (CrossRef Link).

[10] Y. Li, Y. Chen, C. S. Chen, Z. Wang, and Y. Zhu, "Charging while moving: Deploying wireless chargers for powering wearable devices,” IEEE Transactions on Vehicular Technology, vol. 67, no. 12, pp. 11575-11586, December, 2018. Article (CrossRef Link).

[11] Y. Zhang, S. He and J. Chen, "Near optimal data gathering in rechargeable sensor networks with a mobile sink,” IEEE Transactions on Mobile Computing, vol. 16, no. 6, pp. 1718-1729, June, 2017. Article (CrossRef Link).

[12] L. Fu, L. He, P. Cheng, Y. Gu, J. Pan and J. Chen, "ESync: Energy synchronized mobile charging in rechargeable wireless sensor networks," IEEE Transactions on Vehicular Technology, vol. 65, no. 9, pp. 7415-7431, September, 2016. Article (CrossRef Link).

[13] M.H. Anisi, G. Abdul-Salaam, M.Y.I. Idris, A.W.A. Wahab and I. Ahmedy, "Energy harvesting and battery power based routing in wireless sensor networks,” Wireless Networks, vol.23, no.1, pp.249-266, January, 2017. Article (CrossRef Link).

[14] S. Guo, C. Wang and Y. Yang, "Joint mobile data gathering and energy provisioning in wireless rechargeable sensor networks,” IEEE Transactions on Mobile Computing, vol. 13, no. 12, pp. 2836-2852, December, 2014. Article (CrossRef Link).

[15] T. D. P. Perera, D. N. K. Jayakody, S. K. Sharma, S. Chatzinotas, and J. Li, "Simultaneous wireless information and power transfer (SWIPT): Recent advances and future challenges," IEEE Communications Surveys \& Tutorials, vol. 20, no. 1, pp. 264-302, Firstquarter, 2018.

Article (CrossRef Link).

[16] I. Flint, X. Lu, N. Privault, D. Niyato and P. Wang, "Performance analysis of ambient RF energy harvesting with repulsive point process modeling," IEEE Transactions on Wireless Communications, vol. 14, no .10, pp. 5402-5416, October, 2015. Article (CrossRef Link).

[17] N. Van Huynh, D. T. Hoang, X. Lu, D. Niyato, P. Wang, and D. I. Kim, “Ambient backscatter communications: A contemporary survey,” IEEE Communications Surveys \& Tutorials, vol. 20, no. 4, pp. 2889-2922, Fourthquarter, 2018. Article (CrossRef Link). 
[18] X. Ye and W. Liang, "Charging utility maximization in wireless rechargeable sensor networks," Wireless Networks, vol. 23, no. 7, pp. 2069-2081, October, 2017. Article (CrossRef Link).

[19] Y. Shu, H. Yousefi, P. Cheng, J. Chen, Y. J. Gu, T. He and K. G. Shin, "Near-optimal velocity control for mobile charging in wireless rechargeable sensor networks," IEEE Transactions on Mobile Computing, vol. 15, no. 7, pp. 1699-1713, July, 2016. Article (CrossRef Link).

[20] L. Fu, P. Cheng, Y. Gu, J. Chen and T. He, "Optimal charging in wireless rechargeable sensor networks," IEEE Transactions on Vehicular Technology, vol. 65, no. 1, pp. 278-291, January, 2016. Article (CrossRef Link).

[21] X. Li, Q. Tang and C. Sun, "Energy efficient dispatch strategy for the dual-functional mobile sink in wireless rechargeable sensor networks," Wireless Networks, vol. 24, no. 3, pp. 671-681, April, 2018. Article (CrossRef Link).

[22] G. Han, A. Qian, J. Jiang, N. Sun and L. Liu, "A grid-based joint routing and charging algorithm for industrial wireless rechargeable sensor networks," Computer Networks, vol.101, pp. 19-28, June, 2016. Article (CrossRef Link).

[23] H. Salarian, K. W. Chin and F. Naghdy, "An energy-efficient mobile-sink path selection strategy for wireless sensor networks," IEEE Transactions on Vehicular Technology, vol. 63, no. 5, pp. 2407-2419, June, 2014. Article (CrossRef Link).

[24] A. Mehrabi and K. Kim, "Maximizing Data collection throughput on a path in energy harvesting sensor networks using a mobile sink," IEEE Transactions on Mobile Computing, vol. 15, no. 3, pp. 690-704, March, 2016. Article (CrossRef Link).

[25] H. Ju and R. Zhang, "Throughput maximization in wireless powered communication networks," IEEE Transactions on Wireless Communications, vol. 13, no. 1, pp. 418-428, January, 2014. Article (CrossRef Link).

[26] H. Ju and R. Zhang, "User cooperation in wireless powered communication networks," in Proc. of IEEE Global Communications Conf., pp. 1430-1435, December 8-12, 2014. Article (CrossRef Link).

[27] IBM CPLEX Optimizer for z/OS.IBM ILOG CPLEX Optimizer, IBM Corporation, 2013. https://www.ibm.com/analytics/cplex-optimizer

[28] N. Karmarkar, “A new polynomial-time algorithm for linear programming," Combinatorica, vil. 4, no. 4, pp. 373-395, December, 1984. Article (CrossRef Link). 

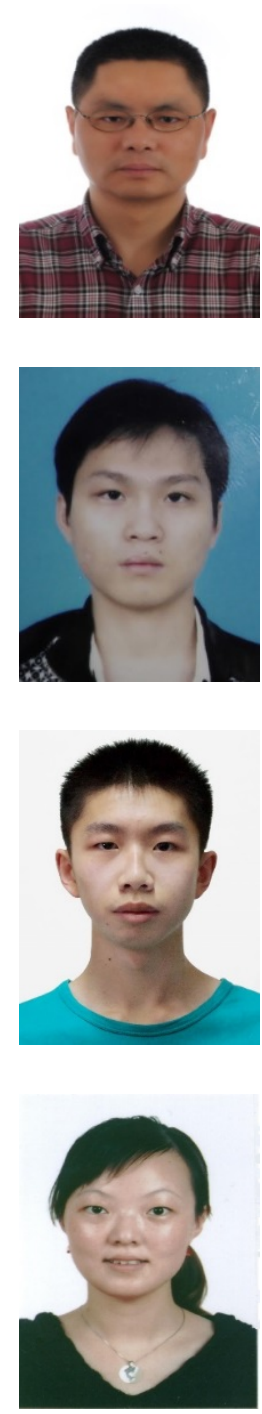

Xianzhong Tian received the B.S. degree in mathematics from Hangzhou Normal University, Hangzhou, China, in 1991; the M.S. degree in computer application technology from Zhejiang University, Hangzhou, China, in 1999; and the Ph.D. degree in control theory and control engineering from Zhejiang University of Technology, Hangzhou, China, in 2013. He is currently a professor with School of Computer Science and Technology, Zhejiang University of Technology, China. His research area includes network coding, wireless sensor networks, and the optimization of protocol for wireless networks.

Jiacun He received his B.Sc. degree in computer science and engineering in 2015 from Zhejiang University of Technology, Hangzhou, China, where he is currently working toward the M.S. degree in computer science and technology. His main research interests include wireless rechargeable sensor networks and mobile computing.

Yuzhe Chen received his B.Eng. degree in internet of things engineering in 2016 from Zhejiang University of Technology, Hangzhou, China, where he is currently working toward the M.S. degree in computer science and technology. His research interests include battery-free networks and internet of things.

Yanjun Li received the B.S. and Ph.D. degrees from Zhejiang University, Hangzhou, China, in 2004 and 2009, respectively, and another Ph.D. degree from Nancy University, Villers-les-Nancy, France, in 2010. She is currently an Associate Professor in School of Computer Science and Technology, Zhejiang University of Technology, Hangzhou, China. Her research area includes protocol and algorithm design in wireless networks. She has published more than 60 referred technical papers in proceedings and journals 